\title{
Quantum contextuality in classical information retrieval
}

\author{
Romàn Zapatrin ${ }^{\mathrm{a}^{*}}$ \\ a Department of Informatics, The State Russian Museum, Inżenernaya 4, 191186, St.Petersburg, Russia \\ *Corresponding author E-mail: Roman.Zapatrin@gmail.com
}

\author{
Article Info \\ Keywords: Quantum entanglement, \\ Non-classical correlation, Information \\ retrieval \\ 2010 AMS: 81P13, 62F15 \\ Received: 12 March 2018 \\ Accepted: 15 April 2018 \\ Available online: 30 June 2018
}

\begin{abstract}
Document ranking based on probabilistic evaluations of relevance is known to exhibit nonclassical correlations, which may be explained by admitting a complex structure of the event space, namely, by assuming the events to emerge from multiple sample spaces. The structure of event space formed by overlapping sample spaces is known in quantum mechanics, they may exhibit some counter-intuitive features, called quantum contextuality. In this Note I observe that from the structural point of view quantum contextuality looks similar to personalization of information retrieval scenarios. Along these lines, Knowledge Revision is treated as operationalistic measurement and a way to quantify the rate of personalization of Information Retrieval scenarios is suggested.
\end{abstract}

\section{The evolution of information needs}

The notion of information needs was clearly formulated by Tailor [12]. Along with the development of IR systems the very structure of information needs, of queries was subject to evolution. Briefly, its mainstream can be described as a transition (read upwards)

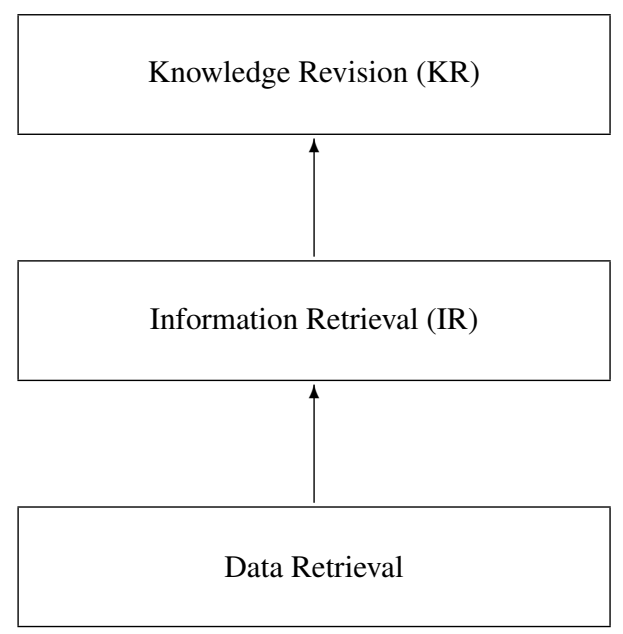

each stage using the previous one as a background. Information Retrieval uses Data Retrieval environment yet modifying the structure of queries, as formulated by Lancaster "An information retrieval system does not inform (i.e. change the knowledge of) the user on the subject of his inquiry. It merely informs on the existence (or non-existence) and whereabouts of documents relating to his request" [5]. Then the next stage is the increasing personalization of search. The user interacts with an IR environment having a goal to update the state of his knowledge (belief) rather than to retrieve a particular document. This way Information Retrieval serves for Knowledge Revision (KR). How quantum mechanics comes? The chain (1.1) can be compared with the transition from classical mechanics, dealing with the absolute character of the values measured, to quantum mechanics, where the result of a measurement is a result of an act of will of an observer rather 
than retrieving a pre-existing value. In both extreme cases, the retrieval act is nothing but a measurement. Similar to the evolution of the notion of measurement, the retrieval metaphors evolve.

We shall deal and with the general notion of Information Needs (IN), ranging them in four levels [12]

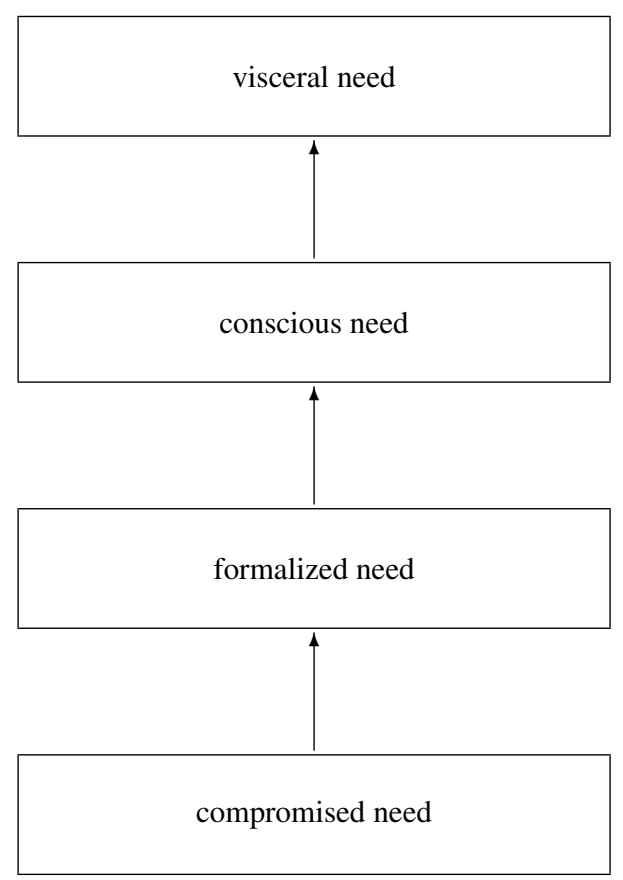

with the following meaning

- The visceral need is the actual, but unexpressed, need for information.

- The conscious need is a within-brain description of the need.

- The formalized need is a formal statement of the question.

- The compromised need is the question as presented to the information system.

The chain (1.1) reflects the upwards transition in the above list, and the personalization tightly approaches to the visceral IN. In this Note I deal with the quantification of personalization - the crucial part of Knowledge Revision - using quantum metaphor. The technical basis for this quantitative approach is formed of the following research lines:

- Simulation of quantum contextuality effects by finite automata and the evaluation of the amount of memory required for this simulation [4]. Our basic idea is to revert this argumentation and to evaluate the features of a quantum system, which can be in certain sense simulated by giver IR environment.

- The evaluations of violations of classical probabilistic laws by index term probabilities, carried out by Melucci [7] and the quantitative evaluation of the amount of contextuality by Svozil [11]

\section{On the nature of non-classical correlations}

In general, non-classical correlations appear when Kolmogorovian probability model is no longer applicable. The basic point of Kolmogorovian model is the existence of a (single) sample space $\Omega$. The events are subsets of $\Omega$, while the points of the sample space are elementary and independent.

In order to test this or that model, we employ Accardi's statistical invariants [2], they allow to test the applicability of Kolmogorovian model. Given:

- a family of discrete maximal observables $\left\{A_{\alpha}: \alpha=1, \ldots T\right\}$ ( $T$ being finite), each observable $A_{\alpha}$ takes the finite number of values $a_{j \alpha}^{(\alpha)}$ labelled by $j_{\alpha}=1, \ldots, n$

- the experimentally measurable conditional probabilities $p_{j_{\alpha}, j_{\beta}}(\beta \mid \alpha)$

$$
p_{j_{\alpha}, j_{\beta}}(\beta \mid \alpha)=P\left(A_{\beta}=a_{j \beta}^{(\beta)} \mid A_{\alpha}=a_{j \alpha}^{(\alpha)}\right)
$$

The problem is: does there exist a probability space $(\Omega ; \mathscr{F} ; P)$ and $T$ measurable partitions $A_{j}^{(\alpha)}$ of cardinality $n$ (the number of distinct values of each observable is assumed to be the same)

$$
A_{j}^{(\alpha)}, \alpha=1, \ldots T, j=1, \ldots n
$$

such that for any $\alpha, \beta=1, \ldots T$ one has

$$
P\left(A^{(\beta)}=a_{j}^{(\beta)} \mid A^{(\alpha)}=a_{i}^{(\alpha)}\right)=\frac{P\left(A_{j}^{(\beta)} \cup A_{i}^{(\alpha)}\right)}{P\left(A_{j}^{(\beta)}\right)}
$$


In order to get the answer, a linear programming problem is to be solved [1], that is, the problem of the existence of a single sample space is finitely decidable.

In the sequel we shall need the special case of three observables $A, B, C$, each taking only two values $a_{1}, a_{2}$ for $A, b_{1}, b_{2}$ for $B$ and $c_{1}, c_{2}$ for the observable $C$. The transition probability matrices for each pair of observables, being bistochastic, each has only one numeric parameter, denote the appropriate matrices as

$$
\begin{aligned}
& P(A \mid B)=P=\left(\begin{array}{cc}
p & 1-p \\
1-p & p
\end{array}\right) \\
& P(B \mid C)=Q=\left(\begin{array}{cc}
q & 1-q \\
1-q & q
\end{array}\right) \\
& P(C \mid A)=R=\left(\begin{array}{cc}
r & 1-r \\
1-r & r
\end{array}\right)
\end{aligned}
$$

then these transition probabilities can be described by a Kolmogorovian model (that is, they are produced by a single sample space) if and only if

$$
|p+q-1| \leq r \leq 1-|p-q|
$$

\section{Melucci operationalistic metaphor}

There is a straightforward analogy between IR and the process of measurement, called Melucci metaphor. There is a search machine, which we may treat to be prepared in certain state, and there is an observer, which performs a measurement. It is typical that the preparation of query system does not assume a query asked by the user, this causes a mismatch, which is to be handled [7].

The situation when a mismatch between the preparation and measurement occurs is a source of paradoxes and counter-intuitive observable consequences of quantum mechanics. It results in the possible randomness of single accounts, though previous stages were deterministically prepared. To deal with it, context translation is introduced as handling the mismatch between state preparation and measurement. In quantum mechanics this metaphorically looks as follows [10]. Suppose an electron is prepared, using Stern-Gerlach device, in pure spin stat along $z$ axis, always showing spin up. Then we decide to ask the so-prepared electron a complementary question: "what is direction of spin along the $x$ axis?" Quantum mechanics tells us that the electron is completely incapable to store more than one bit of information (assuming this is not so leads to direct experimental contradictions). That is why the electron gives a random reply on this query. This is what makes it different from deterministic query agents, who are not able to handle improper input, on which they offer no answer.

Modern IR environments are no longer so rigid, they easily handle any kind of input: if you ask them, almost always you get an answer, but sometimes the relevance of this answer for you personally may be of zero value. To overcome this, search engines are configured to track user's requests, or, in other words, to keep the context associated with particular user and his present role. Altogether, each such particular action I call knowledge revision scenario. In practice this is done by seeding pebbles along the way the user goes through the jungles of World Wide Web, say, by storing browser's cookies. These pebbles are, after all, just sequences of bits. Now suppose our task is to judge to what extent the act of measurement is personalized, let us view it from a perspective of quantum measurement. To do it, recall a series of recent works summarized in [4].

\section{Quantifying the personality in Knowledge Revision scenarios}

In brief, quantum contextuality manifests itself as follows: when measuring quantum systems, the result may depend on which other compatible observables are measured simultaneously. Furthermore, these other observables may be just intended to be measured rather than really measured. This cloud of potentially co-measurable values is referred to as context. When simulating a quantum system by agents with internal memory (recall that, as told above, quantum system are so smart that they behave in this way without having internal memory), the agent will attain different internal states in course of carrying out a sequence of elementary queries. The minimal amount of memory needed to simulate particular manifestations of quantum contextuality is called memory cost of this quantum effect. The paper [4] explores the memory cost of simulating quantum contextuality effect observed on singlet states of positronium. It gives a clue to draw a correspondence:

$$
\text { quantum contextuality } \longrightarrow \text { its memory cost }
$$

In general, the memory cost increases as more and more contextuality constraints are considered. The complexity of contextuality constrains depends, in its turn, on the dimension of the state space of the system in question.

I suggest the following technical idea. The argumentation of the authors of [4] is reverted. We start with an IR environment and ask how complex quantum contextual features it may exhibit? Furthermore, we may reduce the answer to just a number (or a string of numbers), namely, the dimensionality (or a tensor product structure - TPS [13]) of a quantum system demonstrating similar context dependence.

$$
\text { KR scenario } \longrightarrow \text { quantum system }
$$

How to do this? What is to be simulated? Here, I dwell only on the logical and certain probabilistic aspects of simulation. To do it, the proper tools to deal with the structure of the collection of properties of a system are introduced.

Overlapping contexts. It was observed by different authors that complex IR systems are not well described by probabilistic models based on a single sample space. In [9] it was explicitly shown that Bayesian reasoning in its direct for fails and, in order to get adequate evaluations, when writing conditional probabilities $P(A \mid B)$ one should take care about specifying the context - a particular sample space, in which these conditional probabilities are calculated. In the meantime, the small sample spaces are not separated - thy overlap, there are events belonging to different contexts. It occurs that the classical contingency table 


\begin{tabular}{|l|c|c|c|}
\hline & RETRIEVED & NOT RETRIEVED & \\
\hline RELEVANT & $A \cap B$ & $A \cap \bar{B}$ & $A$ \\
NON-RELEVANT & $\bar{A} \cap B$ & $\bar{A} \cap \bar{B}$ & $\bar{A}$ \\
\hline & $B$ & $\bar{B}$ & \\
\hline
\end{tabular}

ceases to be adequate. The reason is that even within a single scenario both $A$ and $B$ may belong to different contexts, in particular, $\bar{A}$ is no longer uniquely defined by $A$ (the same to $B$ and $\bar{B}$ ). How to capture this structure? A tool of combinatorial nature is needed to describe overlapping contexts. First note that a single sample space is structureless, all its elements are equally (un)related with each other. In case of overlapping contexts this is no longer the case. A graphical (and combinatorial) way to capture such relations was suggested by R.Greechie (see [3] for an overview). The idea is to

(i) consider all the elements of all sample spaces together

(ii) label each element with a tag pointing to appropriate context

The Kolmogorovian probabilities (and hence Bayesian inference) come from the fact that the logic of statements about the appropriate sample space is Boolean. In case of pasted contexts this is no longer so, the structure of all the statements about the IR environment is no longer Boolean.

How contextuality effects come? Mainly, in the form of Kochen-Specker reasoning stating that particular hypothetical probability assignments do not exist such as a total probability distribution on the whole diagram viewed as a single sample space. The consequence of such results is signaling that the evaluation of conditional probabilities based on standard Bayes model will be no loner adequate. For examples of such violations in quantum mechanics see [4], in IR this also takes place, see, for instance [7]. Quantitatively it looks as follows.

'How much contextuality'? So far, only qualitative ideas were provided. The next step is to try to evaluate them, putting the question 'How much contextuality'? A possible transparent answer was recently proposed in [11]. We take a representative sampling of observables, and simply check the ratio of the triples, for which Accardi inequalities (2.4) are violated.

Using the ideas of [11], the rate of personalization can be evaluated in a similar way. First, by random sampling, triples of properties, that is, yes-no queries are picked. Then, for each triple, the transition probability matrices (2.3) are calculated. For each particular sample triple the inequalities (2.4) are checked. Then the ratio of samples is calculated:

$$
\text { Pers }=\frac{\text { number of triples violating }(2.4)}{\text { total number of sampled triples }}
$$

\section{Conclusions}

Vector models of IR become more and more popular, first of all because they make it possible to carry out multi-document actions. In this paper I dwell on a QIA framework [8]. The basic ingredient of QIA framework is a Hilbert space $\mathscr{H}$ called the information need space. In its simplest form, IN space is linear space of elementary (atomic) topics. In my approach, I suggest to start introducing the IN space to satisfy the necessary amount of capturing contextuality. The ideology of IN space is the closest to that of quantum mechanics. In QM, the state space of a system is a space of some internal (in the deepest possible sense) features of a system, while the observables are expressed in terms of operators and other derived structures on the state space. Similar things happen in QIA approach. The space of information needs exists per se, we may treat it as spanned on elementary entities, but this will be nothing, but a representation of this space. The source of emergence of this space lies in the multicontextual structure described in the previous section. Furthermore, as pointed in [6], [7], the correlations, which occur in IR environment may even be stronger than quantum ones. In this case a straightforward Hilbert space model may fail to work properly, and we may call 'foil quantum theories ${ }^{1}$ " to grip these situations.

So far, I was interested in information retrieval situations, when the result of a particular action may depend on other actions, which the IR agent could in principle do alongside with the actions actually performed. This phenomenon is called contextuality, we encounter it in IR, we have to take it into account, to work with it. A similar kind of dependence takes place in quantum mechanics.

\begin{tabular}{l|l|l} 
Quantum Mechanics & Information Retrieval \\
contextuality & $\leftrightarrow$ & personalization
\end{tabular}

The difference is that in QM contextuality appears by itself, not being originated by some 'internal mechanisms'. The situations where contextuality occurs depend on the state space of the system the structure of observables involved. In the realm of QM we can quantitatively evaluate the rate of contextuality [11]. The origin of contextuality effects in IR stems from personalization of query scenarios. The personalization, in turn, can be quantified by memory resources required to keep tracking the information needs of a particular user (note that 'user' in this context might not be a single person, nor even a 'person' at all). The idea of this Note was to demonstrate that using quantum mechanics formalism, we can quantify the rate of personalization in particular IR environments. To do this, I suggest to reverse the procedure of estimation of memory cost of quantum contextuality based on simulating quantum systems by finite automata. Instead, a KR scenario (which as a matter of fact is a sequence of queries upon a finite automaton) is suggested to be simulated by appropriate scenario of quantum measurement, demonstrating the same contextuality features. As a result, a Hilbert space of appropriate quantum system emerges together with a collection of observables. This Hilbert space is suggested to play the role of information need space, which is developed within QIA (quantum information access) framework for Information Retrieval. Technically, the IN space is built starting from Greechie-like diagrams (pasted overlapping contexts, see Section 4 above, capturing the particular IR environment. QIA framework provides more flexible machinery to deal with information needs than any classical probabilistic approach by that simple reason that it incorporates the latter. But

${ }^{1}$ Mathematically rigorous constructions, describing ways the world could have been were it not quantum mechanical 
we should be aware that it is not ultimately general. In quantum realm, we have non-classical correlations and the present state of our knowledge shows that quantum mechanics is enough to explain all them. However, IR may in principle provide stronger-than-quantum correlations. For them, 'foils of quantum theory' - the operational theories, which do not compete with quantum mechanics, but generalize it to the extent not demanded in modern physics [6, 14], these theories may be of help in Information Processing.

Acknowledgments. I greatly appreciate Cris Calude, Karl Svozil and Jozef Tkadlec for stimulating discussions on quantum contextuality during my stay in Technical University of Vienna, supported by the Ausseninstitut and the Institute of Theoretical Physics of the Vienna University of Technology. A financial support from Russian Basic Research Foundation (grant 10-06-00178a) is appreciated.

\section{References}

[1] L. Accardi and Fedullo A. On the statistical meaning of complex numbers in quantum mechanics. Il Nuovo Cimento, 34(7):161-172, October 1982.

[2] Luigi Accardi. Axioms for quantum probability. In Quantum Probabilty, volume 73 of Banach Center Publications, page 1. Polish Academy of Sciences, 2006.

[3] R. J. Greechie and S. P. Gudder. Quantum logics. In C.A. Hooker, editor, Contemporary Research in the Foundations and Philosophy of Quantum Theory, pages 143-173. Reidel, Dordrecht, 1973.

[4] Matthias Kleinmann, Otfried Guehne, Jose R. Portillo, Jan-Åke Larsson, and Adan Cabello. Memory cost of quantum contextuality. New Journal of Physics, 13:113011, 2011.

[5] F.W. Lancaster. Information Retrieval Systems: Characteristics, Testing and Evaluation. Wiley, New York, 1968.

[6] Yeong-Cherng Liang, Robert W. Spekkens, and Howard M. Wiseman. Specker's parable of the overprotective seer: a road to contextuality, nonlocality and complementarity. Phys. Rep., 506(1-2):1-39, 2011.

[7] Massimo Melucci. When Index Term Probability Violates the Classical Probability Axioms Quantum Probability can be a Necessary Theory for

[8] Benjamin Piwowarski, Ingo Frommholz, Mounia Lalmas, and Keith van Rijsbergen. Exploring a multidimensional representation of documents and queries. In Proceedings of RIAO, 2010.

[9] Stephen Robertson. On event spaces and probabilistic models in information retrieval, presented at. In SIGIR 2002 Workshop on Mathematical/Formal Methods in Information Retrieval, 2004.

[10] Karl Svozil. Quantum information via state partitions and the context translation principle. Journal of Modern Optics, 51:811-819, 2004.

[11] Karl Svozil. How much contextuality? Natural Computing, Online First(nn):nn-nn, 2012.

[12] Robert S. Taylor. Process of asking questions. American Documentation, 13:391-396, October 1962.

[13] Paolo Zanardi. Virtual quantum subsystems. Phys.Rev.Lett., 87:077901, 2001.

[14] Roman Zapatrin. Factory of realities: on the emergence of virtual spatiotemporal structures. In: "Beyond Peaceful Coexistence: The Emergence of Space, Time and Quantum”, Ignazio Licata, Ed., World Scientific (2016), pp. 201-220, 2016. 